\title{
PRINCIPAL CONGRUENCES IN DE MORGAN ALGEBRAS
}

\author{
by M. E. ADAMS* \\ (Received 7th February 1986)
}

\section{Introduction}

A congruence relation $\theta$ on an algebra $L$ is principal if there exist $a, b \in L$ such that $\theta$ is the smallest congruence relation for which $(a, b) \in \theta$. The property that, for every algebra in a variety, the intersection of two principal congruences is again a principal congruence is one that is known to be shared by many varieties (see, for example, $\mathrm{K}$. A. Baker [1]). One such example is the variety of Boolean algebras. De Morgan algebras are a generalization of Boolean algebras and it is the intersection of principal congruences in the variety of de Morgan algebras that is to be considered in this note.

A de Morgan algebra is an algebra $(L ; \vee, \wedge, \sim, 0,1)$ of type $(2,2,1,0,0)$ such that $(L ; \vee, \wedge, 0,1)$ is a distributive $(0,1)$-lattice, $\sim$ is a dual $(0,1)$-lattice endomorphism (so that $\sim(a \vee b)=\sim a \wedge \sim b, \sim(a \wedge b)=\sim a \vee \sim b, \sim 0=1$, and $\sim 1=0)$, and $\sim \sim a=a$. A de Morgan algebra is a Kleene algebra providing the inequality

$$
a \wedge \sim a \leqq b \vee \sim b
$$

holds.

Let $\mathbf{B}, \mathbf{K}$ and $\mathbf{M}$ denote the varieties of Boolean, Kleene, and de Morgan algebras, respectively. It was shown by J. A. Kalman [10] that the lattice of non-trivial varieties of de Morgan algebras is a 3-element chain $\mathbf{B} \subset \mathbf{K} \subset \mathbf{M}$.

The question of whether the intersection of two principal congruences of a de Morgan algebra is again principal was first asked by H. P. Sankappanavar [14]. The problem was restated by T. S. Blyth and J. C. Varlet [3] in their study of principal congruences in $M S$-algebras. ( $M S$-algebras are a generalization of de Morgan and Stone algebras and, as was shown by K. A. Baker (see H. Lasker [11]), the variety of Stone algebras has the above property.) The purpose of this note is to give an example of a de Morgan algebra for which the intersection of two of its principal congruences fails to be principal (see Section 3) and to show that no such Kleene algebra may be found (see Theorem 9 of Section 4).

It is a pleasure to acknowledge conversations on this topic with T. S. Blyth.

\section{Preliminaries}

For further information and references on de Morgan algebras, the reader is referred to R. Balbes and Ph. Dwinger [2] or M. S. Goldberg [8].

*The support of the NSF (grant \# 8402909) is gratefully acknowledged. 
A topological duality for distributive $(0,1)$-lattices was given by $\mathbf{H}$. A. Priestley and extended to de Morgan algebras by W. H. Cornish and P. R. Fowler. Sections 3 and 4 are based on this duality. A brief outline of the basic requirements follows. (A fuller account of topological duality may be found in either of the survey papers of B. A. Davey and D. Duffus [7] or H. A. Priestley [13].)

Let $P$ be a partially ordered set and, for $Q \subseteq P$, let $(Q]$ and $[Q)$ denote the order ideal and filter generated by $Q$. Then $Q$ is decreasing, increasing, or convex providing $Q=(Q]$, $Q=[Q)$, or $Q=(Q] \cap[Q)$, respectively. For partially ordered sets $P$ and $P^{\prime}$, a mapping $\phi: P \rightarrow P^{\prime}$ is order-preserving if $\phi(x) \leqq \phi(y)$ whenever $x \leqq y$.

Given a topology $\tau$ defined on a partially ordered set $P$, the pair $(P, \tau)$ is called an ordered space. The space is totally order-disconnected provided, for $x, y \in P$ with $x \leq y$, there exists a clopen decreasing $Q \subseteq P$ such that $y \in Q$ and $x \notin Q$. If $(P, \tau)$ is a compact totally order-disconnected space, then it is called a Priestley space. Let $T$ denote the category of all Priestley spaces together with all continuous order-preserving maps. As shown in H. A. Priestley [12], the category of distributive $(0,1)$-lattices with $(0,1)$-lattice homomorphisms is dually equivalent to $\mathbf{T}$. If, under this duality, $L$ and $(P, \tau)$ are associated, then the elements of $L$ correspond to the clopen decreasing subsets of $P$. Further, if $f: L \rightarrow L^{\prime}$ is associated with the continuous order-preserving map $\phi: P^{\prime} \rightarrow P$, then $f(a)=b$ iff $\phi^{-1}(A)=B$ where $A$ and $B$ are the clopen decreasing subsets that represent $a$ and $b$. Inspection shows that $f$ is onto iff $\phi$ is an order-isomorphism. Then, since congruences correspond to onto $(0,1)$-lattice homomorphisms, it follows that the congruences of a distributive $(0,1)$-lattice $L$ are in a one-to-one correspondence with the closed subsets of $P$ or, as is more convenient in the present context, the (complementary) open subsets of $P$. In which case, for a congruence $\theta$ associated with an open set $Q$

$$
a \equiv b(\theta) \text { iff }((A \backslash B) \cup(B \backslash A)) \subseteq Q
$$

where $a$ and $b$ are represented by $A$ and $B$. In particular, the lattice of congruence relations on $L$ is isomorphic to the lattice of open subsets of $P$ (where both are ordered by inclusion).

Since de Morgan algebras are distributive $(0,1)$-lattices, they should be dually equivalent to some suitable category of Priestley spaces. Let $\mathbf{T}_{M}$ denote the category whose objects $(P, \tau, \zeta)$ are Priestley spaces endowed with a continuous anti-isomorphism $\zeta$ such that $\zeta^{2}(x)=x$ for all $x \in P$ (to be referred to as $m$-spaces) and whose morphisms are those continuous order-preserving maps $\phi: P \rightarrow P^{\prime}$ for which $\phi \circ \zeta(x)=\zeta \circ \phi(x)$ (to be referred to as m-maps).

Proposition 1 (W. H. Cornish and P. R. Fowler [5]). The category of de Morgan algebras is dually equivalent to the category $\mathbf{T}_{M}$.

If $L$ and $(P, \tau, \zeta)$ correspond under this duality, then, for $a \in L, \sim a$ is represented by $P \backslash \zeta^{-1}(A)$ where $A$ represents $a$. If an onto homomorphism $f: L \rightarrow L^{\prime}$ is associated with an $m$-map $\phi: P^{\prime} \rightarrow P$, then $\phi$ is an order isomorphism that also preserves $\zeta$. Thus, congruences of $L$ correspond to open subsets of $P$ that are closed under $\zeta$ (i.e., open sets $Q \subseteq P$ such that $Q=\zeta(Q)$ ). In particular, the lattice of congruences of a de Morgan 
algebra is isomorphic to the lattice of open subsets of the associated $m$-space that are closed under $\zeta$ (see W. H. Cornish and P. R. Fowler [6]).

The next two lemmas characterize those open sets that correspond to principal congruences under the duality.

Lemma 2. Let $L$ be a de Morgan algebra with associated $m$-space $(P, \tau, \zeta)$. For $a \leqq b \in L$, let $\theta(a, b)$ (the principal congruence generated by $a$ and $b$ ) be represented by the open set $Q \subseteq P$. Then, for some convex clopen set $Q^{\prime}, Q=Q^{\prime} \cup \zeta\left(Q^{\prime}\right)$.

Proof. Let $Q^{\prime}=B \backslash A$. Certainly, $Q^{\prime}$ is a convex clopen set. Moreover, since any de Morgan congruence is also a lattice congruence, it follows from the above remarks that $Q \supseteq Q^{\prime}$. But $Q^{\prime} \cup \zeta\left(Q^{\prime}\right)$ is the smallest open set to contain $B \backslash A$ and be closed under $\zeta$; that is $Q=Q^{\prime} \cup \zeta\left(Q^{\prime}\right)$.

Lemma 3. Let $L$ be a de Morgan algebra with associated $m$-space $(P, \tau, \zeta)$. If, for an open set $Q \subseteq P$, there exists a clopen convex set $Q^{\prime}$ such that $Q=Q^{\prime} \cup \zeta\left(Q^{\prime}\right)$, then $Q$ represents a principal congruence on $L$.

Proof. $Q^{\prime}$ closed implies that $\left(Q^{\prime}\right]$ is closed. Further, since $Q^{\prime}$ is also open, it follows that $\left(Q^{\prime}\right] \backslash Q^{\prime}$ is closed. The compact total order-disconnectedness of $(P, \tau)$ then yields a clopen decreasing set $A$ such that .

$$
\left(Q^{\prime}\right] \backslash Q^{\prime} \subseteq A \text { and } \quad Q^{\prime} \cap A=\emptyset
$$

Let $B=A \cup Q^{\prime}$. Then, since $Q^{\prime}$ is clopen and convex, $B$ is a clopen decreasing set as well as $A$. If $a$ and $b$ are the elements of $L$ that are represented by $A$ and $B$, respectively, then, as in Lemma 3 , the principal congruence $\theta(a, b)$ corresponds to the open set $(B \backslash A) \cup \zeta(B \backslash A)$. But, by construction, $Q^{\prime}=B \backslash A$ (and, hence, $Q$ represents a principal congruence).

In Section 4 , we shall need to recognize those $m$-spaces that correspond to Kleene algebras. Clearly, since every Kleene algebra is a de Morgan algebra, they are dually equivalent to some subcategory of $\mathbf{T}_{M}$. Let $\mathbf{T}_{K}$ denote the full subcategory of $\mathbf{T}_{M}$ whose objects (to be referred to as $k$-spaces) $(P, \tau, \zeta)$ satisfy $x$ is comparable to $\zeta(x)$ for every $x \in P$.

Proposition 4 (W. H. Cornish and P. R. Fowler [5]). The category of Kleene algebras is dually equivalent to $\mathbf{T}_{K}$.

\section{Intersection of principal congruences in de Morgan algebras}

The object of this section is to give an example of a de Morgan algebra for which two principal congruences have a non-principal intersection. Consider the following:

Let $L$ be the de Morgan algebra associated with the $m$-space $(P, \tau, \zeta)$ where $P$ is the ten-element $\operatorname{set}\left\{x_{0}, x_{1}, x_{2}, x_{3}, x_{4}, \zeta\left(x_{0}\right), \zeta\left(x_{1}\right), \zeta\left(x_{2}\right), \zeta\left(x_{3}\right), \zeta\left(x_{4}\right)\right\}, \tau$ is discrete, and the order relation is as shown in Figure 1. 


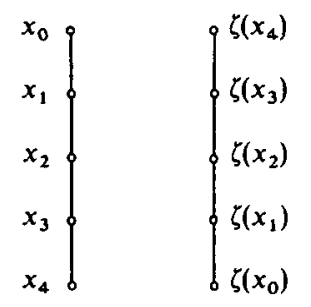

Figure 1.

Clearly, as suggested, $(P, \tau, \zeta)$ is an $m$-space. It remains to identify two principal congruences the intersection of which is not.

Let $Q_{0}^{\prime}=\left\{x_{0}, x_{1}, x_{2}, \zeta\left(x_{4}\right)\right\}$. Then $Q_{0}^{\prime}$ is convex and so, by Lemma $3, Q_{0}=Q_{0}^{\prime} \cup \zeta\left(Q_{0}^{\prime}\right)=$ $P \backslash\left\{x_{3}, \zeta\left(x_{3}\right)\right\}$ represents a principal congruence $\theta_{0}$.

Let $Q_{1}^{\prime}=\left\{x_{2}, x_{3}, x_{4}, \zeta\left(x_{0}\right)\right\}$. Then $Q_{1}^{\prime}$ is also a convex set and, hence, $Q_{1}=$ $Q_{1}^{\prime} \cup \zeta\left(Q_{1}^{\prime}\right)=P \backslash\left\{x_{1}, \zeta\left(x_{1}\right)\right\}$ represents a principal congruence $\theta_{1}$.

Since the lattice of congruences on $L$ is isomorphic to the lattice of subsets of $P$ that are closed under $\zeta, \theta=\theta_{0} \wedge \theta_{1}$ is associated with

$$
Q=Q_{0} \cap Q_{1}=\left\{x_{0}, x_{2}, x_{4}, \zeta\left(x_{0}\right), \zeta\left(x_{2}\right), \zeta\left(x_{4}\right)\right\} .
$$

By Lemma 2, if $Q$ represents a principal congruence, then there exists a convex set $Q^{\prime} \subseteq Q$ such that $Q=Q^{\prime} \cup \zeta\left(Q^{\prime}\right)$. However, any convex $Q^{\prime} \subseteq Q$ contains at most one element of $\left\{x_{0}, x_{1}, x_{2}, x_{3}, x_{4}\right\}$ and at most one element of $\left\{\zeta\left(x_{0}\right), \zeta\left(x_{1}\right), \zeta\left(x_{2}\right), \zeta\left(x_{3}\right), \zeta\left(x_{4}\right)\right\}$. Thus, for any such $Q^{\prime}, Q^{\prime} \cup \zeta\left(Q^{\prime}\right)$ contains at most four elements and, in particular, $Q^{\prime} \cup \zeta\left(Q^{\prime}\right) \neq Q$. We conclude that $\theta$ is not principal.

\section{Intersection of principal congruences in Kleene algebras}

The purpose of this section (Theorem 9) is to show that, for a Kleene algebra, the intersection of two principal congruences is always a principal congruence.

As observed in W. H. Cornish and P. R. Fowler [6], any $k$-space $(P, \tau, \zeta)$ is the union of subspaces

$$
\vec{P}=\{x \in P: \zeta(x) \leqq x\} \quad \text { and } \quad \overleftarrow{P}=\{x \in P: x \leqq \zeta(x)\} .
$$

The space $\vec{P}$ and $\overleftarrow{P}$ are closed, homeomorphic, order anti-isomorphic, and, respectively, increasing and decreasing. They intersect in $\{x \in P: \zeta(x)=x\}$ which is a (discretely ordered) Boolean space contained in the minimal points of $\vec{P}$.

The following is an immediate consequence of the above:

Lemma 5. For a $k$-space $(P, \tau, \zeta)$, let $X \subseteq P$ be closed under $\zeta$. If, for $Y \subseteq X$, $X \cap \vec{P}=Y \cap \vec{P}$, then $X=Y \cup \zeta(Y)$.

Lemma 6. For a $k$-space $(P, \tau, \zeta)$, let $X=Y \cup \zeta(Y)$ where $Y \subseteq P$ is a convex set. Then $X \cap \vec{P}$ is convex. 
Proof. Suppose, for $x, y, z, \in P, x<y<z$ where $x, z \in X \cap \vec{P}$. It is to be shown that $y \in X \cap \vec{P}$.

Since $x \in \vec{P}$,

$$
\zeta(z)<\zeta(y)<\zeta(x) \leqq x<y<z
$$

It follows from the definition of $X$ that

$$
x \text { or } \zeta(x) \in Y \text { and } z \text { or } \zeta(z) \in Y \text {. }
$$

If $z \in Y$, then $y \in Y$. Otherwise, $\zeta(z) \in Y$ and, hence, $\zeta(y) \in Y$. In either case, $y \in X$. But, by hypothesis, $y \in \vec{P}$.

The following technical consequence of Lemma 6 will be required in the proof of Lemma 8.

Lemma 7. For a $k$-space $(P, \tau, \zeta)$, let $X=Y \cup \zeta(Y)$ where $Y \subseteq P$ is a convex set. Then

$$
((X \cap \vec{P}] \backslash X] \cap(X \cap \vec{P} \cap \stackrel{\leftarrow}{P})=\emptyset
$$

Proof. Suppose otherwise and choose $x \in((X \cap \vec{P}] \backslash X] \cap(X \cap \vec{P} \cap \overleftarrow{P}) \neq \emptyset$. Then $x \in X \cap \vec{P}$ and $x \leqq y$ for some $y \in(X \cap \vec{P}] \backslash X$. In turn, $y \leqq z$ for some $z \in X \cap \vec{P}$. Thus, $x \leqq y \leqq z$ where $x, z \in X \cap \vec{P}$. By Lemma $6, y \in X \cap \vec{P}$ which contradicts the fact that $y \notin X$.

Lemma 8. For a $k$-space $(P, \tau, \zeta)$ and convex clopen $Q^{\prime} \subseteq P$, there exists a convex clopen $Q^{\prime \prime} \subseteq Q$ (where $\left.Q=Q^{\prime} \cup \zeta\left(Q^{\prime}\right)\right)$ such that $Q^{\prime \prime} \cap \vec{P}=Q \cap \vec{P}$.

Proof. We must exhibit a suitable candidate for $Q^{\prime \prime}$. In order to do so we will construct a clopen convex set $X$ such that $X$ is closed under $\zeta$,

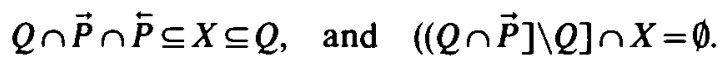

To begin with we shall aim for less and find a set $Y$ that satisfies each of the conditions required of $X$ except the last.

Suppose $x \in(P \backslash Q) \cap \vec{P}$. Then $x \leq y$ for any $y \in Q \cap \vec{P} \cap \overleftarrow{P}$. Since $Q \cap \vec{P} \cap \overleftarrow{P}$ is closed, there exists a clopen increasing set $Y_{x}$ such that $x \in Y_{x}$ and $Y_{x} \cap(Q \cap \vec{P} \cap \overleftarrow{P})=\emptyset$. The sets $Y_{x}$ form a cover of the closed set $(P \backslash Q) \cap \vec{P}$. Choose, by compactness, a finite subcover and denote its union by $Y^{\prime}$. Thus $Y^{\prime}$ is a clopen increasing set such that $(P \backslash Q) \cap \vec{P} \subseteq Y^{\prime}$. Consequently, $\zeta\left(Y^{\prime}\right)$ is a clopen decreasing set for which $(P \backslash Q) \cap \bar{P} \subseteq \zeta\left(Y^{\prime}\right)$. Since both $Y^{\prime}$ and $\zeta\left(Y^{\prime}\right)$ have empty intersection with $Q \cap \vec{P} \cap \stackrel{\leftarrow}{P}, Y=P \backslash\left(Y^{\prime} \cup \zeta\left(Y^{\prime}\right)\right)$ is a clopen convex

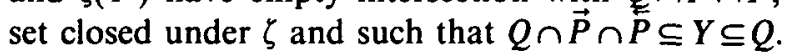

We now find $X$.

The set $((Q \cap \vec{P}] \backslash Q]$ is a closed decreasing set. By Lemma 7 , for $x \in((Q \cap \vec{P}] \backslash Q]$, there exists a clopen decreasing set $Z_{x}$ such that $x \in Z_{x}$ and $Z_{x} \cap(Q \cap \vec{P} \cap \vec{P})=\emptyset$. Choose a finite subcover of $((Q \cap \vec{P}] \backslash Q]$ and denote its union by $Z^{\prime}$. Then $Z^{\prime}$ is a clopen decreasing set such that

$$
((Q \cap \vec{P}] \backslash Q] \subseteq Z^{\prime} \quad \text { and } \quad Z^{\prime} \cap(Q \cap \vec{P} \cap \overleftarrow{P})=\emptyset
$$


Let $Z=P \backslash\left(Z^{\prime} \cup \zeta\left(Z^{\prime}\right)\right)$. It follows that $Z$ is a clopen convex set closed under $\zeta$ such that

$$
(Q \cap \vec{P} \cap \overleftarrow{P}) \subseteq Z \text { and }((Q \cap \vec{P}] \backslash Q] \cap Z=\emptyset .
$$

Then $X=Y \cap Z$ has the required properties

Let $Q^{\prime \prime}=X \cup(Q \cap \vec{P})$.

Clearly, $Q^{\prime \prime} \subseteq Q$ and, from $Q \cap \vec{P} \subseteq Q^{\prime \prime} \subseteq Q$, it follows that $Q^{\prime \prime} \cap \vec{P}=Q \cap \vec{P}$.

To see that $Q^{\prime \prime}$ is clopen, observe that, since $X$ is clopen, $(Q \cap \vec{P}) \backslash X$ is closed. On the other hand, $Q \backslash \overleftarrow{P}$ open implies that $(Q \backslash \tilde{P}) \backslash X=(Q \cap \vec{P}) \backslash X$ is open. Thus, $Q^{\prime \prime}=X \cup((Q \cap \vec{P}) \backslash X)$ is the union of two clopen sets.

It remains to show that $Q^{\prime \prime}$ is convex. Consider $x<y<z$ where $x, z \in Q^{\prime \prime}$. There are two cases:

Suppose $x \in Q \cap \vec{P}$. Then $z \in Q \cap \vec{P}$ as well. By Lemma $6, y \in Q \cap \vec{P}$ and, since $Q \cap \vec{P}=$ $Q^{\prime \prime} \cap \vec{P}, y \in Q^{\prime \prime}$.

Alternatively, suppose $x \notin Q \cap \vec{P}$. Then $x \in X$. For $z \in X, y \in Q^{\prime \prime}$ follows from the convexity of $X$. Assume therefore that $z \notin X$ (and, hence, $z \in Q \cap \vec{P}$ ). Either $y \in \vec{P}$ or $y \in \bar{P} \backslash \vec{P}$. Suppose first that $y \in \vec{P}$. If $y \notin Q$, then $y \in(Q \cap \vec{P}] \backslash Q$. In which case $(y] \cap X=\emptyset$. Since this contradicts the choice of $x \in X$, we conclude that $y \in Q$. Thus, $y \in Q \cap \vec{P}$ and so, as before, $y \in Q^{\prime \prime}$. Suppose therefore $y \in \overleftrightarrow{P} \backslash \vec{P}$. Then $x<y$ implies $x<y<\zeta(y)<\zeta(x)$. Since $X$ is closed under $\zeta, \zeta(x) \in X$. But $X$ is convex and so $y \in Q^{\prime \prime}$.

Theorem 9. For a Kleene algebra, the intersection of two principal congruences is a principal congruence.

Proof. Let $L$ be a Kleene algebra with associated $k$-space $(P, \tau, \zeta)$. For $i=0,1$, let $Q_{i} \subseteq P$ correspond to the principal congruence $\theta_{i}$. Since the lattice of congruences on $L$ is isomorphic to the lattice of open subsets of $P$ that are closed under $\zeta, \theta_{0} \wedge \theta_{1}$ is represented by the set $Q_{0} \cap Q_{1}$.

For $i=0,1$, choose $Q_{i}^{\prime \prime}$ as in Lemmas 2 and 8 . Then

$$
\left(Q_{0} \cap Q_{1}\right) \cap \vec{P}=\left(Q_{0}^{\prime \prime} \cap Q_{1}^{\prime \prime}\right) \cap \vec{P}
$$

By Lemma 5,

$$
Q_{0} \cap Q_{1}=\left(Q_{0}^{\prime \prime} \cap Q_{1}^{\prime \prime}\right) \cup \zeta\left(Q_{0}^{\prime \prime} \cap Q_{1}^{\prime \prime}\right)
$$

Because the intersection of two clopen convex sets is a clopen convex set, it follows from Lemma 3 that $Q_{0} \cap Q_{1}$ corresponds to a principal congruence.

\section{REFERENCES}

1. K. A. BAKer, Primitive satisfaction and equational problems for lattices and other algebras, Trans. Amer. Math. Soc. 190 (1974), 125-150.

2. R. Balbes and Ph. Dwinger, Distributive Lattices (University of Missouri Press, Columbia, Missouri, 1974). 
3. T. S. Blyth and J. C. Varlet, Congruences on MS-algebras, Bull. Soc. Roy. Sci. Liège 53 (1984), 341-362.

4. S. Burris and H. P. Sankappanavar, A Course in Universal Algebra (Springer-Verlag, New York, 1981).

5. W. H. Cornish and P. R. Fowler, Coproducts of de Morgan algebras, Bull. Austral. Math. Soc. 16 (1977), 1-13.

6. W. H. Cornish and P. R. Fowler, Coproducts of Kleene algebras, J. Austral. Math. Soc. Ser. A 27 (1979), 209-220.

7. B. A. Davey and D. Duffus, Exponentiation and duality, in Ordered Sets (ed. I. Rival), NATO Advanced Study Institutes Series (D. Reidel, Dordrecht, 1982), pp. 43-96.

8. M. S. Goldberg, Distributive p-algebras and Ockham Algebras; A Topological Approach (Ph.D. Thesis, La Trobe University, Australia, 1979).

9. G. Grätzer, Lattice Theory: First Concepts and Distributive Lattices (Freeman, San Francisco, California, 1971).

10. J. A. Kalman, Lattices with involution, Trans. Amer. Math. Soc. 87 (1958), 485-491.

11. H. Lakser, Principal congruences of pseudocomplemented distributive lattices, Proc. Amer. Math. Soc. 37 (1973), 32-36.

12. H. A. Priestley, Representation of distributive lattices by means of ordered Stone spaces, Bull. London Math. Soc. 2 (1970), 186-190.

13. H. A. Priestley, Ordered sets and duality for distributive lattices, Ann. Discrete Math. 23 (1984), 39-60.

14. H. P. Sankappanavar, A characterization of principal congruences of de Morgan algebras and its applications, in Mathematical Logic in Latin America (ed. A. I. Arruda, R. Cuaqui and N. C. A. da Costa) (North-Holland, Amsterdam, 1980), pp. 341-349.

STATE UNIVERSITY OF NEW YORK

New PALTZ

NEW YORK 12561

U.S.A. 